\title{
When ECAD meets MCAD - the design pattern of innovation
}

\author{
WANG Jing ${ }^{1, a}$, LIU Ting-ting ${ }^{2, b}$ \\ ${ }^{1}$ Chongqing College of Electronic Engineering, Chongqing 401331, China \\ ${ }^{2}$ Chongqing College of Electronic Engineering, Chongqing 401331, China \\ aemail: wangjingad09@126.com , bemail: loveancient@foxmail.com
}

Keywords: Electronic CAD, Machinery CAD, 3D model, Synchronous design

\begin{abstract}
When the electronic computer aided design (ECAD) meets the mechanical computer aided design (MCAD), the process, method and scheme of the design in the new electronic products will have a qualitative leap, and the three-dimensional (3D) design is one of the best ways to realize the improvement of new production quality. 3D design could accurately express the engineer's design ideas, according with engineers' thinking mode and design habits. In the design of new products, engineers could import the three-dimensional model of the electronic products to MCAD software, then mechanical designers are expected to observe the appearance comprehensively and objectively, design its mechanical, structure and shell. Experimental results indicate that the proposed method could predict the actual effect of the new product in advance, avoiding the mismatch between the electronic products and the mechanical casing. Also it will greatly improve the design efficiency and design quality, achieving the synchronous design of the electronic and mechanical products and actualize the integration of design and manufacture.
\end{abstract}

\section{Introduction}

Imagine, if you had already got the expensive PCB (printed circuit board) from the manufacturer, but then you find that it did not fit into the shell. How disappointed you are and how much time and energy you have to spend to redesign this?

Currently, in the design of new electronic products, the internal structure includes the compact collocation of electronic components and machinery parts which could coordinate routine work. Integrated mechanical and electrical design elements in the work is a complex and multi-step process, Altium Designer could provide assistance in the integration of these two aspects of the data, therefore the electronic designers and mechanical designers could work faster, easier and their collaboration could become more useful. Altium Designer provides advanced mechanical computer aided design MCAD (Machine Computer Aided Design) and ECAD (Electronic Computer Aided Design ) data exchange function, Allows ECAD designers to import, process, and check the Mechanical elements of the PCB design; it can also import the electronic products of three-dimensional map to mechanical CAD software. Mechanical designers need to design the mechanical, structural, shell aspects, in order to achieve the synchronization of electronic and mechanical design. Through this way, electronic designers and mechanical designers could find their communication more convenient, therefore they could make a more comprehensive, careful, and reasonable design, largely shorten the development cycle of new products ${ }^{[1-3]}$.

\section{Fusion of ECAD and MCAD}

In some companies, the electronic and mechanical design has been executed by different teams in the process of implementation of the task design, mechanical and electronic design engineer their use their own application software to complete the design task, if their exchanges are not fully convenient, does not reach the designated position, it is possible to make the design of new products into the waste; and now the new products, electronic and mechanical interaction more and more across the two fields work together to design a high quality in line with customer demand for new products, it is very important, when the ECAD met MCAD, the problem is solved, and three dimensional design ECAD and MCAD integration provides reliable guarantee.

In the past, three dimensional designs could be carried out in the mechanical design software. Currently, the use of three-dimensional design technology in the electronic CAD software is also very 
convenient. The 3D solid design system has become the main design method of the mechanical and electronic product development personnel. It is an important tool in the design process. It embodies the design idea of 3D design ${ }^{[4]}$.

Product design is the process of using the 3D design system on the computer to continue to grow, and eventually become a complete design. In other words, the design of the 3D model on the computer have the advantage of high degree of visualization, the 3D model allows the designer to observe and examine the design of the product model, the 3D model is highly interactive, the designer can be drag, rotate and modify the 3D model at any time. Without 3D model which could be used to observe and examine, or the model is difficult to interact and modify the conditions, Designer can only rely on experience and imagination to design. The 3D solid design system with high degree of visualization and interaction makes it feasible to build 3D model in real time. This real-time interactive 3D modeling process has changed the design method of the traditional "thinking", which makes the product design process more interactive, to reach kind of thinking, building the 3D model, and the optimizing method. This kind of thinking, building the 3D model, and the optimizing method could allow designers to make more attempts "If put it in this way, how?" try, Designer makes a more comprehensive consideration of the design and thus design a better product ${ }^{[5]}$. The advantages of three-dimensional design is beyond doubt, Directly in the 3D environment for product development and design is the trend, and is the important method to design the high quality and high performance products.

\section{The establishment of 3D model}

Considering the support of Altium Designer to 3D, the shape of components could be modeled using Altium Designer 3D Body object. The establishment of 3D model of the PCB board is introduced by a case study in this paper.

Designer need to use Altium software to design the motor drive circuit PCB board and material after welding components, as shown in figure 1. The establishment of 3D model of PCB board was introduced on the 2 components of PCB board.

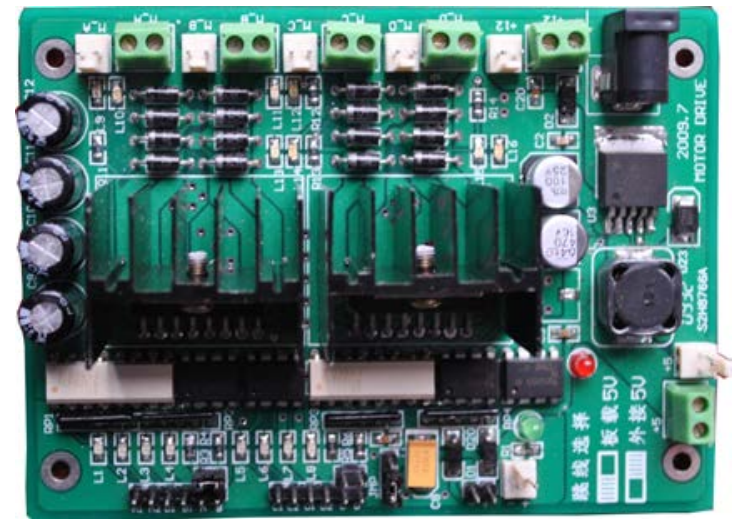

Fig. 1 PCB board for motor drive circuit

\subsection{Establishment of 3D model of the light emitting diode (LED)}

In Altium Designer software, execute the Place 3D Body command, the pop Fig. 2 dialog box, draw a cylinder.

(1) The Cylinder is selected in Figure 2 the 3D Model Type region.

(2) In the Properties, select the level of the 3D model, where the top layer is selected.

(3) In the display, select the color of the 3D model, in this choice and in kind similar to the color "red".

(4) In the cylinder, the radius of the cylinder is $1.5 \mathrm{~mm}$, the height of the cylinder is $4 \mathrm{~mm}$, the Standoff Height is $0 \mathrm{~mm}$, the rotating angle of the cylinder along the direction of $\mathrm{X}, \mathrm{Y}$ and $\mathrm{Z}$ is $0^{\circ}$.

The 3D Model Management dialog is shown in Figure 2. After setting up, the 3D model can be placed in the position of the light emitting diode of the PCB board ${ }^{[6]}$. 


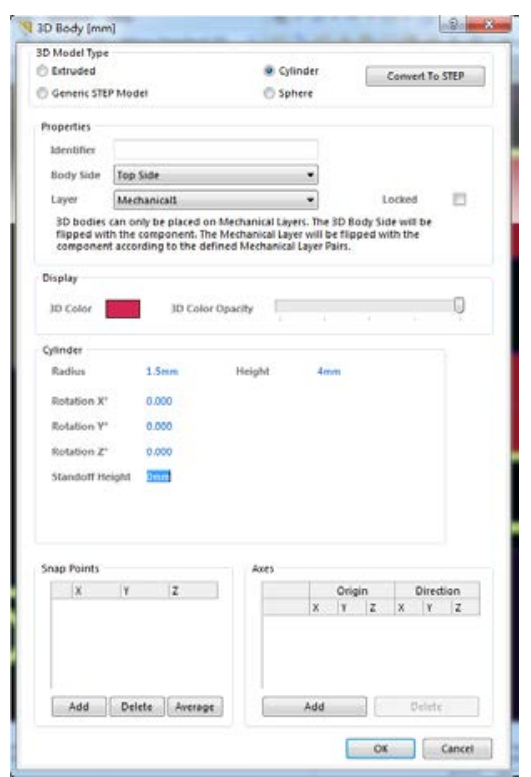

Fig.2 Draw cylinder dialog box

Draw the LED semi sphere, drawing method with cylinder, the following 2 different.

(1) Sphere is chosen for the 3D Model Type region shown in Figure 2.

(2) In the Sphere column, select the radius of the ball to $1.5 \mathrm{~mm}$, Standoff Height of the ball to $4 \mathrm{~mm}$.

After setting up, the 3D model can be placed in the position of the light emitting diode of the PCB board, draw a good 3D model of the light emitting diode as shown in figure 3.

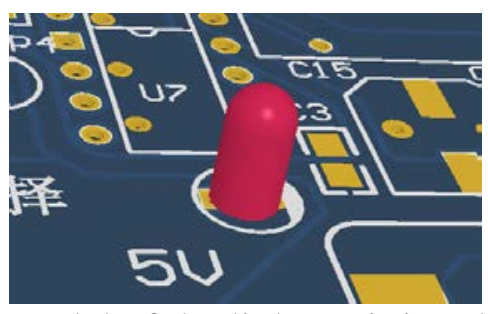

Fig.3 3D model of the light emitting diode

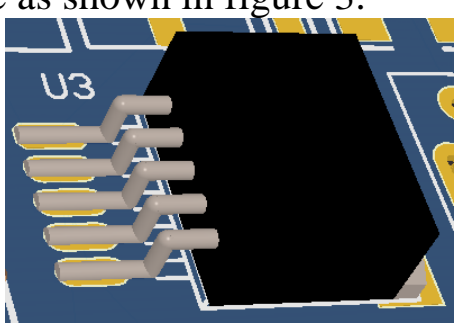

Fig.4 Regulator 3D model

\subsection{D model to establish the regulator}

Execute Place 3D Body command, draw the gray rectangle.

(1) The 3D Model Type region in Figure 2 is selected for the Extruded.

(2) In the Properties, select the level of the 3D model, where the top layer is selected.

(3) In the display, select the color of the 3D model, in this choice and in kind similar to the color "gray".

(4) In the Extruded column, select the overall Height for the 1mm, select Standoff Height for the $0 \mathrm{~mm}$.

After setting up, you can design 3D model of the irregular rectangle of the regulator in the U3 board of PCB, the gray irregular rectangle of the regulator is Length:21mm; wide:3mm; high: 1mm; angle: $30^{\circ}$, as shown in figure 4 .

Draw a black rectangle with the same as above. In the Extruded column, select the overall Height for $4.5 \mathrm{~mm}$, select Standoff Height for 0 $\mathrm{mm}$. After setting up, you can draw the 3D model of the regulator's black rectangle in the PCB board's U3, as shown in Figure 4. Black rectangle is Length: 21mm, width: $17 \mathrm{~mm}$, height: $4.5 \mathrm{~mm}$.

Execute Place 3D Body command and draw two horizontal cylinders from right to left.

Methods with the light emitting diode, the Radius of the cylinder is $0.4 \mathrm{~mm}$, the Height of the cylinder is $2.5 \mathrm{~mm}$, the Standoff Height is $2 \mathrm{~mm}$, the Rotation of the cylinder along the direction of $\mathrm{X}$, $\mathrm{Y}$ and $\mathrm{Z}$ is $90^{\circ}$.

After setting up, the 3D model can be placed in the PCB board of the regulator.

The dimensions of the other cylinder are as follows: 
The Radius of the cylinder is $0.4 \mathrm{~mm}$, the Height of the cylinder is $4 \mathrm{~mm}$, the Standoff Height of the cylinder is $0 \mathrm{~mm}$, the cylinder is $90^{\circ}$ in the Rotation of $\mathrm{X}, \mathrm{Y}, \mathrm{Z}$ direction.

Draw a vertical cylinder, the size is as follows:

The Radius of the cylinder is $0.4 \mathrm{~mm}$, the Height of the cylinder is $2 \mathrm{~mm}$, the Standoff Height of the cylinder is $0 \mathrm{~mm}$, the cylinder is $0^{\circ}$ in the Rotation of $\mathrm{X}, \mathrm{Y}, \mathrm{Z}$ direction.

The pins of the 3 cylinders are connected with the sphere (from top to bottom, from right to left respectively with three spheres).

The dimensions of the sphere are as follows:

In the Sphere column, the Radius of the Sphere is $0.4 \mathrm{~mm}$, the Standoff Height of the Sphere is $2 \mathrm{~mm}$; the Radius of the sphere is $0.4 \mathrm{~mm}$, the Standoff Height of the Sphere is $0 \mathrm{~mm}$.

Other pins are copied, pasted is $\mathrm{OK}$, and the $3 \mathrm{D}$ model of the regulator is plotted as shown in figure 4.

Note: the first and the second Sphere to be placed with the vertical cylinder overlap placed in figure 4.

Using the basic method described above, we can draw the 3D model of any complex components; draw the 3D model of the motor drive circuit of all components on the PCB board, as shown in figure 5. In this model, the 3D model can be compared with that of Figure 1, they are so similar.

\section{Export and import data}

In order to pass the design data between the electronic CAD and the mechanical CAD design environment, we adopt the STEP file. STEP's full name is Standard for Exchange of Product model data, STEP standards for the CAD/CAM system is to provide neutral product data and the development of public resources and application model, which involves the construction, engineering, structure, mechanical, electrical, electronic engineering and hull structure, etc. STEP is the standard file format for most of the mechanical CAD tools, which have been widely used in many engineering applications. Data from the Altium Designer can be used directly in the mechanical CAD software or elsewhere. STEP format can be used to accurately to set up highly complex model objects, and could be used in the production of mechanical structure near perfect data check.

Export PCB 3D model document for STEP file, Export steps are as follows:

(1) Executive menu commands: File $\rightarrow$ Save As or File $\rightarrow$ Save Copy As, Save As dialog will appear.

(2) Select a suitable location and name for the file to be exported, from the Save as type list to select the STEP Export.

(3) Click the Save button to appear in the Export Options STEP dialog, for deciding which objects to output. Note that the circuit board itself will always be exported.

(4) The STEP Export Options dialog box is used to determine which objects are included in the output file:

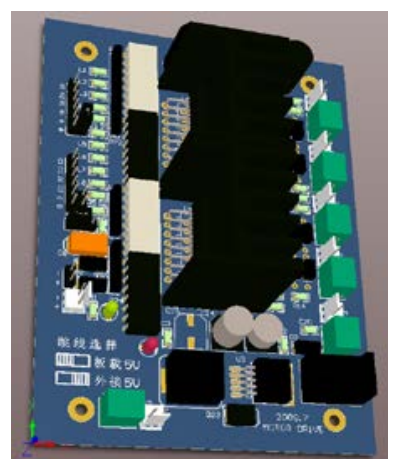

Fig.5 3D model of PCB board for motor drive circuit

Components with 3D Bodies regional options for 3D body are selected components of the printed circuit board and other free floating 3D body. If you only need to export the circuit board, make sure that you don't have any objects on the PCB, and then click the Export Selected option. 
3D Bodies Export Options regional options are used when the element 3D body model contains both the STEP model and the simple 3D model, with the purpose to decide which model to derive.

Prefer Simple bodies will export a simple 3D model, if the element contains a simple 3D body model and contains the STEP model.

Prefer STEP models will export the STEP model, if the element contains a simple 3D body model and contains the STEP model.

Export both simple bodies \& STEP models will export a simple 3D and STEP models. If you have only a simple 3D model, then it will always be exported.

Pad Holes region provides an option to export all over the hole.

(5) Above set up according to the need to choose a good, you can complete the export.

Now it can be directly opened PCB 3D step file in the Pro/ENGINEER software. start the software, open the step file, display the motor drive circuit of the 3D model is not complete, press the $\AA^{\circ}$ button on the toolbar to display the full 3D model, as shown in figure 6 .

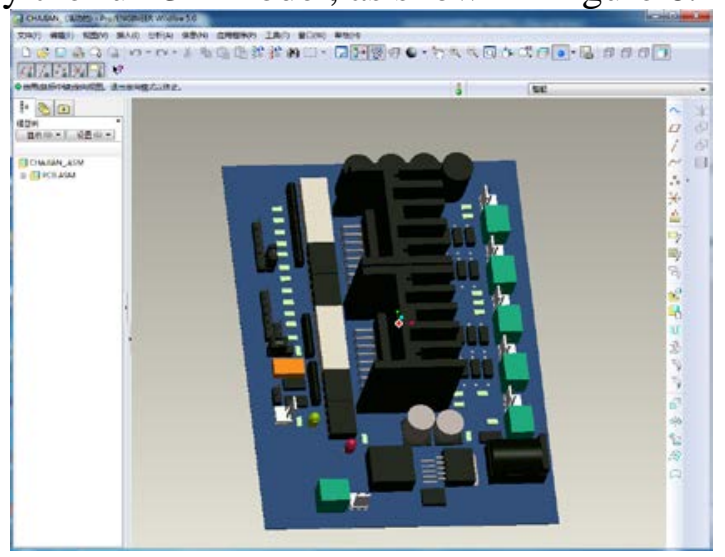

Fig.6 Altium Designer software design of 3D PCB map into the Pro/Engineer software

In the Pro/E software, you can use the following control view.

Rotate: drag the mouse button.

Translation: Shift key + mouse button, move the mouse.

Zoom: Ctrl key + mouse button, the mouse up and down to move.

Flip: Ctrl key + mouse button, the mouse around mobile.

Quick zoom: rotate the mouse button roller.

Rotation center settings: When rotating graphics, open the center switch of the system toolbar, rotate the center icon as the center of rotation; Turn the center switch off the system toolbar, and rotate the center of the mouse cursor at the position of the view ${ }^{[7-8]}$.

Now it will be able to easily carry out the design of the mechanical, structural, shell, etc. of the PCB board in the Pro/E software, to avoid the PCB board and the mechanical casing does not match the situation, resulting in a complete ECAD from MCAD to 3D solution.

Pro/E、UG、CATIA、Solidworks several mainstream 3d design software can open a step file, this is not to say, by readers according to their own needs.

AutoCAD can also open the Step file, but can only display the two-dimensional, cannot display three-dimensional.

\section{Conclusion}

Through establishing 3D model of PCB board in Altium software, converting to "step" file, and then imported it into Pro/E software, it could be used to design the product's mechanical, structural, shell and other aspects. Experimental results indicate that the scheme could ensure that in the primary stage of design process, electronic designers and mechanical designers could synchronize their design process, the 3D PCB board designed by electronic designer can be imported into 3D mechanical design software, so does the mechanical, structural, and shell of the PCB board; mechanical designer design Machinery, structure, shell and other models can also be imported into 
the PCB software, to ensure that the design of the PCB board can be properly loaded into the machine, and it also could ensure the confliction between the electronic design and mechanical design could be settled, improve products' design quality, to foresee the actual effect of the new product on the computer. Through the integration of machinery and electronics to promote the innovation and continuous improvement of new products and with the synchronized development of mechanical and electronic design, we could achieve the early settlement of the integration of machinery and electronics, shorten the development cycle of new products, and accelerate the pace of new products $^{[9-10]}$. When ECAD meets MCAD, we could realize the double harvest between the design cycle and the design quality and to accomplish the integration of design and manufacturing.

\section{References}

[1] Li Wenshi, Qian min, Huang Qiuping. Three dimensional microelectronics review [J].Microelectronics,2004,(03):227-230.doi:10.3969/j.issn.1004-3365.2004.03.003.

[2] ROSELINE BENIERE,GERARD SUBSOL,GILLES GESQUIERE et al. A comprehensive process of reverse engineering from 3D meshes to CAD models[J].Computer-Aided Design,2013,45(11):1382-1393.

[3] PUREVDORJ NYAMSUREN,SOO-HONG LEE,SUNGMIN KIM et al. A Web-Based Revision Control Framework for 3D CAD Model Data[J].International Journal of Precision Engineering and Manufacturing,2013,14(10):1797-1803.

[4] Guo Qinglin. Application of 3D CAD technology in the design of mechanical industry [J]. Electronic technology and software engineering, 2015,01: 98

[5] China Wikipedia. Beyond the entity modeling - interactive combination of parts [DB/OL]. http://www.chinabaike.com/t/30167/2014/0903/2833624.html

[6] Wang Jing, Liu Tingting. Altium Designer 2013 Case tutorial [M]. Beijing: China Water\&Power Press , 2014.

[7] Du Baishi, Yang Fuzeng, Design and analysis of Pro/E planetary gear mechanism [M]. Beijing: Publishing House Of Electronics Industry,2014.

[8] Wang Keyin, Yan Wuping. Pro/ENGINEER Wildfire 5.0 Chinese version of industrial design case real station[M]. Beijing: China Machine Press,2011.

[9] CIMdata product reviews. PTC of ECAD-MCAD collaboration solutions " integration of mechanical product design and electronic products design"[EB/OL]. http://www.docin.com/p-51120376.html.2009-04.

[10]Rob Evans. ECAD-MCAD collaborative design - a mechanism for change [DB/OL]. [2015-11-03].http://www.docin.com/p-888496 